\title{
Infant Formula
}

National Cancer Institute

\section{Source}

National Cancer Institute. Infant Formula. NCI Thesaurus. Code C81249.

A nutritional substitute for breast milk, usually with a foundation of cow or soy milk. 